CRÍTICA, Revista Hispanoamericana de Filosofia

Vol. XXII, No. 66 (diciembre 1990): 25-37

\title{
MÁS ALLÁ DE LA PRESUPOSICIÓN NEWTONIANA: PROPIEDADES GENUINAMENTE DISPOSICIONALES EN LA MECÁNICA CUÁNTICA
}

SERGIO MARTinEZ

Instituto de Investigaciones Filosóficas Universidad Nacional Autónoma de México

1. A partir de la revolución científica del siglo XVII, el desarrollo de la física en particular, y en gran medida de las ciencias naturales en general, ha traído consigo la elaboración de una idea que ha servido como guía metodológica central: la estructura de los sistemas materiales que nuestras teorías buscan explicar es "externa" a un nivel ontologicamente fundamental de partículas (o puntos espacio-temporales como es el caso en las teorías de campo). Según este principio metodológico, si la descripción de un proceso físico incluye condiciones iniciales que no son independientes, $i$. $e$. que están funcionalmente relacionadas entre sí, esto implica que el proceso no ha sido descrito cabalmente. Este principio metodológico, al que llamaremos principio metodológico newtoniano, se ha desarrollado en íntima relación simbiotica con la siguiente tesis metafísica que, de manera programática, podemos formular como sigue: la estructura física de un sistema compuesto es la suma de las propiedades de sus subsistemas. Esta presuposición ontológica acerca de la relación entre el todo y las partes la llamaremos la presuposición merológica newtoniana. Después de hacer ver de manera breve el origen y significado de esta presuposición en la física moderna, y de dar algunos ejemplos de cómo ésta se 
cuestiona actualmente en diferentes teorías de la física, paso ha examinar con más detalle la única teoría ampliamente confirmada de la física en la que se niega la presuposición merologica newtoniana, la mecánica cuántica. Quiero hacer ver cómo en la mecánica cuántica la presencia de una estructura merológica no newtoniana (holista) es el resultado de la presencia de propiedades disposicionales genuinas. Las propiedades disposicionales genuinas de un sistema son propiedades que no pueden reducirse a propiedades ocurrentes (presentes o futuras) de los subsistemas. En la terminología de J. L. Mackie, las propiedades disposicionales genuinas serían las propiedades que no tienen una base categorica.

2. En la física moderna la acción de las fuerzas se describe por medio de ecuaciones diferenciales. Los sistemas físicos sobre los que las fuerzas actúan se identifican en cada instante por medio de sus "estados" o, más bien, por medio del conjunto de valores de las magnitudes que describen ese estado (ver la sección 3). Por ejemplo, las magnitudes de la posición y el momento identifican el tipo de sistemas físicos conocidos como partículas clásicas puntuales. Los valores de las magnitudes de estado funcionan como condiciones iniciales para la determinación de la solución de la ecuación diferencial que permite la predicción de cualquier estado futuro del sistema (suponiendo que las fuerzas se describen por medio de funciones "bien portadas" y que el sistema puede aislarse del medio ambiente con la debida precisión).

La ausencia de una estructura interna, pertinente para la explicación del comportamiento de los sistemas sobre los que actúan las fuerzas, se expresa en el lenguaje de las ecuaciones diferenciales diciendo que en una descripción completa del fenómeno (o clase de fenómenos o procesos) "las condiciones iniciales pueden escogerse arbitrariamente". Esta idea puede reformularse en términos de la noción çe la independencia de magnitudes: un conjunto de magnitudes de un sistema 
es independiente si cualquier combinación posible de valores de las diferentes magnitudes es físicamente posible. Por ejemplo, en la descripción de una partícula clásica la posición y el momento son magnitudes independientes. La idea de independencia de valores de magnitudes es crucial para la descripción de procesos físicos en términos de fases. Un espacio de fase puede pensarse como el espacio producto de las magnitudes de estado.

En tanto que se asume que la estructura causal de un sistema está codificada en las ecuaciones diferenciales que describen la evolución de los sistemas en el tiempo, se asume también que toda estructura causal es dinámica, esto es, se asume que toda explicación del comportamiento de los sistemas físicos puede formularse en términos de su comportamiento en el tiempo. Ésta es una presuposición acerca de la estructura del mundo físico que quizá no sea correcta. Es posible que haya alguna estructura "pertinente" para la explicación de un proceso físico que no pueda expresarse únicamente en términos de las propiedades ocurrentes a través de la evolución dinámica. En este caso, nuestro principio metodológico newtoniano nos llevaría a tratar de "desenmarañar" las condiciones iniciales en casos en que eso no es posible físicamente. Como veremos, esta posibilidad es digna de tomarse muy en serio.

En el ensayo titulado "Organisms as Causal Systems that are Not Mechanisms", Robert Rosen (Rosen 1985) nos habla de la idea básica que guía su trabajo en biofísica. Según Rosen, la mayoría de las teorías de la física moderna tratan exclusivamente de un tipo muy especial de sistemas a los que él llama sistemas simples o mecanismos. Los sistemas simples de Rosen son precisamente aquellos que pueden representarse como trayectorias en un espacio de fase, esto es, para los que es posible desenmarañar las condiciones iniciales. Así, Rosen nos dice que

[I] he mathematical image that Newtonian mechanics gives us 
of a family of structureless particles acted upon by forces is as follows. Such a system is represented by a manifold of posible phases (configurations plus velocities) on which a set of equations of motion, representing forces is superimposed... All this, by the way, is completely preserved in the transition to quantum physics, the only novelty (though it is a major one) resides in the replacement of the Newtonian phases by a more general space of states, related in a much more complicated way with what we actually measure (p. 183).

Por el contrario, los modelos mattmáticos de la organización biológica que Rosen propone no asumen que las magnitudes que caracterizan al estado de un sistema sean independientes unas de otras y del medio ambiente. En particular, Rosen sostiene que algún tipo de causalidad final tiene que reintroducirse en la ciencia para desarrollar un modelo más apropiado de la relación entre el todo y las partes y del papel que desempeña esta relación en la explicación de fenómenos biológicos. Independientemente de la manera como escojamos remplazar el principio metodológico newtoniano, el trabajo de Rosen me parece particularmente interesante como ilustración de un programa de investigación que asocia explícitamente el rechazo del principio metodológico newtoniano con el rechazo de la presuposición merológica implícita. Sus modelos explícitamente rechazan la idea de que las propiedades de un sistema sean explicables totalmente en términos de las propiedades de los subsistemas. La organización biológica no es el resultado, sino el punto de partida para la explicación de la relación entre la biología y la física.

Es importante hacer notar, sin embargo, que Rosen está equivocado cuando piensa que la presuposición newtoniana no es cuestionada en la física moderna y que incluso "la transición a la mecánica cuántica preserva totalmente el marco newtoniano". Por un lado, en la cosmología moderna hay una serie de propuestas teóricas que explícitamente tratan de rechazar la presuposición newtoniana. Penrose (en Penrose 1989), por 
ejemplo, después de hacer un examen de los diversos intentos recientes por incorporar los efectos cuánticos en una teoría satisfactoría de la cosmología nos dice que:

...this separation into dynamical equations and boundary conditions has been historically of vital importance. The fact that it is possible to make such a separation at all es a property of the particular type of equations (differential equations) that always seem to arise in physics. But I do not believe that this is a division that is here to stay. (p. 352)

Por otro lado, si bien en la interpretación usual de la mecánica cuántica parece que la presuposición newtoniana se mantiene porque confunde dos tipos de estado diferentes, en una interpretación que distingue entre los diferentes tipos de estado requeridos para la descripción de un proceso cuántico la presuposición newtoniana tiene que abandonarse, como veremos a continuación. ${ }^{1}$

En el resto de este artículo quiero hacer ver cómo el cuestionamiento de la presuposición newtoniana en la mecánica cuántica lleva a una explicación del holismo cuántico en términos de propiedades genuinamente disposicionales de un sistema compuesto. Estas propiedades describen una estructura que no es reducible a la suma de las propiedades ocurrentes de los subsistemas de un sistema compuesto. En efecto, esta descripción del holismo cuántico hace ver que los sistemas cuánticos son "complejos" (no simples) en el sentido de Rosen.

3. Partimos de la suposición de que todos los sistemas físicos pueden caracterizarse por un conjunto definido y finito de magnitudes (relativos a una teoría). Una magnitud consiste en un conjunto de valores, uno de los cuales, cuando más, es el caso

1 Ver Kochen 1985, Healey 1989, Martínez 1991. En Martínez 1988 y 1990 examino los diferentes conceptos de estado que se requieren para describir un proceso cúntico y hago ver el papel que juegan en la interpretación de la teoría. 
en un momento dado $t .^{2} \mathrm{El}$ valor de la magnitud describe una propiedad del sistema en el tiempo $t$.

Una situación experimental con respecto a una magnitud $M$ es una situación física en la que la magnitud tiene un valor determinado. En la mecánica clásica hay una sola situación experimental físicamente pertinente, pero en la mecánica cuántica hay diferentes situaciones experimentales. Una situación experimental puede en principio determinar más de una magnitud, pero para simplificar la exposición, en lo sucesivo identificaremos situaciones experimentales con magnitudes máximas. Es posible que una magnitud tenga un valor determinado en una situación experimental pero que no lo tenga en otra situación experimental. Como, precisamente, deben entenderse físicamente las situaciones experimentales anteriormente descritas, depende de una serie de decisiones acerca de la interpretación de la teoría cuántica que no nos conciemen en este trabajo. Por ahora es suficiente hacer notar que, dependiendo de como entendemos una situación experimental, los estados que representan los sistemas individuales serán representados por diferentes tipos de filtros en el retículo que representa al sistema. ${ }^{3}$ Los estados individuales describen las propiedades

2 Todo conjunto exclusivo de valores (cuando más denumerable), en el sentido anteriormente mencionado, genera un retículo booleano atómico en el que los átomos corresponden a los valores posibles de la magnitud. Un retículo booleano es un retículo distributivo con un único complemento. Dado un elemento $x$ de un retículo $L$ (con 0 y 1 ), $x$ es complemento de $x^{\prime}$ si $x \wedge x^{\prime}=0$ y $x \vee x^{\prime}=1$. Un complemento $x^{\prime}$ de $x$ es booleano si $x^{\prime}$ y $x$ pertenecen a un mismo retículo booleano. El complemento booleano de $x$ se representa por $x^{\perp}$. En la nota 3 se resumen otras definiciones de la teoría de retículos que utilizamos en este trabajo.

3 Un retículo (finito) puede definirse como un conjunto parcialmente ordenado $L$ por medio de una relación < en el que para todo par de elementos $x, y \in L$ existe una cota superior mínima y una cota inferior máxima. Una cota superior de $\{x, y\}$ es un elemento $z \in L$ tal que $x<z$ y $y<z$. Una cota superior mínima se representa por $x \vee y$. Una cota inferior de un par de elementos $\{x, y\} \in L$ es un elemento $w \in L$ tal que $w<x$ y $w<y$. Una cota inferior máxima de $\{x, y\}$ se representa por $x \wedge y$. Decimos que 
(valores de las magnitudes) que el sistema tiene en una situación experimental dada. Los elementos del filtro y sus complementos booleanos son las propiedades que físicamente se interpretan como si fueran (o no) el caso. Esto es, los elementos del filtro que representa el estado del sistema describen las propiedades cuyo valor de verdad está determinado por la situación experimental. Identificamos estas propiedades que son o no el caso con las propiedades ocurrentes del sistema (ver Martínez 1990). Diremos que un estado individual es del tipo booleano dades del sistema. Por ejemplo, en el retículo representado por medio de un diagrama de orden en la Fig. 1 el estado de tipo $B$ generado por el átomo $a$ en la magnitud $X$ (al que representamos por $[a, X)$ ) es el conjunto de propiedades $\left\{a, b^{\perp}, c^{\perp}, 1\right\}$. Otro ejemplo de un estado de tipo $B$ es $\left\{c, d^{\perp}, f^{\perp}, 1\right\}=[c, Y)$, el estado generado por $c$ en la magnitud $Y$. Un diagrama de orden es una representación gráfica de la relación de cobertura para elementos del retículo (véase nota 3). Asumimos aquí, por razones que se presntan en otros trabajos, que los filtros booleanos describen los estados del sistema (véase Martínez 1991 por ejemplo).

un elemento $x$ cubre un elemento $y(y<x)$ si $y<x$ y no hay un elemento $z$ tal que $y<z<x$. La relación de cobertura se representa en los diagramas de orden por medio de segmentos de recta. Si $y<x$ entonces $y$ está en el extremo inferior del segmento y $x$ en el extremo superior del segmento.

Un filtro (reticular) $F$ es por definición un conjunto de elementos de un retículo $L$ que satisface las condiciones siguientes:

i) si $x, y \in F$ entonces $x \wedge y \in F$;

ii) si $x \in F$ entonces $x \vee y \in F$ para todo $y \in L$.

Un filtro booleano es un filtro cuyos elementos satisfacen la condición de que todos sus elementos pertenecen a un mismo subretículo booleano máximo. Un retículo booleano $B$ es máximo (en un retículo $L$ ) si no hay otro retículo booleano $B^{\prime}$ (en $L$ ) tal que $B$ es una subestructura (subconjunto) de $B^{\prime}$. 


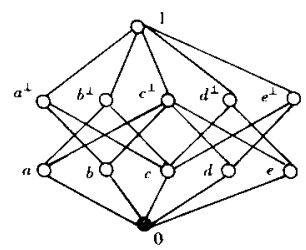

Fig. 1

El diagrama en la Fig. 1 describe un retículo generado por dos magnitudes (retículos booleanos) $X$ y $Y$ que comparten algunos elementos $(c, c, 0,1)$.

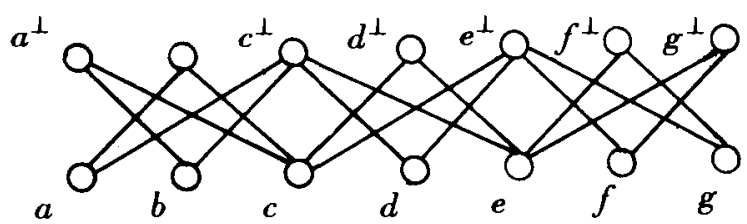

Fig. 2

En la Fig. 2 tenemos el diagrama de un retículo con tres magnitudes máximas representadas por $X, Y$ y $Z$. Decimos que el retículo es de tres dimensiones ya que el número máximo de átomos independientes (de valores diferentes posibles) es de tres. Para simplificar la representación gráfica, no se incluyen las relaciones de cobertura del tipo $0<x$ y $y<1$. El retículo describe un fragmento de la estructura de las posibles propiedades de un sistema físico $S .{ }^{4}$ Veamos cómo.

En primer lugar, un contexto experimental determina que uno de los estados (filtros) de tipo $B$ es el caso. Digamos que este estado inicial está dado por el filtro $[a, X)=\left\{a, b^{\perp}, c^{\perp}, 1\right\}$. Según la mecánica cuántica, si el sistema interactúa con otro sistema, el sistema puede cambiar de situación experimental. En nuestro diagrama asumimos que la situación experimental estaba representada por $X$. Después de una interacción con

$4 L$ un fragmento del retículo $L\left(H_{3}\right)$, el retículo formado por los subespacios cerrados de un espacio de Hilbert de tres dimensiones. 
otro sistema es posible que el sistema entre en la situación $Y$ o en la situación $Z$, o en alguna de las otras situaciones que no están representadas en el diagrama. Es importante hacer notar que si inicialmente el estado del sistema $S$ era $[a, X)$, entonces propiedades como $d$ y $e$, por ejemplo, no tienen un valor de verdad determinado por el estado inicial. Pero, si después de una interacción, la situación experimental es $Y$, entonces $e$ y $f$ tendrán un valor de verdad, $i$. $e$. ellas o su negación (complemento booleano) serán propiedades ocurrentes del sistema $S$.

Supongamos que el retículo parcialmente representado en la Fig. 2 describe la estructura de los estados posibles de un sistema compuesto por dos subsistemas $U$ y $V$. Supongamos además que la propiedad $d$ es la propiedad que puede describirse como la siguiente correlación de propiedades de los subsistemas $U$ y $V$ : "el valor de la magnitud $M$ de $U$ es +1 y el valor de la magnitud $M$ de $V$ es -1 "; y que la propiedad e del sistema compuesto describe la siguiente correlación de propiedades entre los subsistemas $U$ y $V$ : "el valor de la magnitud $M$ de $U$ es -1 y el valor de la magnitud $M$ de $V$ es +1 . $d$ y $e$ describen los posibles resultados de medir una magnitud con sólo dos valores $(+1,-1)$ sujeta a una ley de conservación que diga que los valores de las magnitudes en los subsistemas tiene que sumar $1 .^{5}$

Supongamos que decidimos medir $U$ y encontramos que el valor es +1 , entonces el estado del sistema en relación con la magnitud $M$ tiene que ser descrito por $d$ (i.e. el valor de $M$ en $V$ es -1 con certeza). Antes de medir $M$ en $U$, sin embargo, nuestro estado inicial era $a$ en una situación experimental (que determinaba) $A$ y no $B . d$ no era el caso, ni $d^{\perp}$ era el caso. $c^{\perp}$ era el caso. Pero $c^{\perp}$ dice que, o bien la correlación descrita por

5 En Martínez 1990 hago ver como una situación típica del tipo EPR puede representarse por medio de un retículo en el cual la situación hipotética aquí descrita se modela físicamente. 
$d$ o bien la correlación descrita por $e$ es el caso, pero no hay ninguna especificación adicional de acuendo con la mecánica cuántica. Todo lo que podemos afirmar antes de hacer una medición que nos lleva de una situación $X$ a una situación $Y$ es que si llegamos a una situación $Y$, o bien $d$ o e es el caso. Cual de las dos posibilidades resulta, no lo predice la teoría. $c^{\perp}$ es una propiedad disposicional del sistema compuesto $S$ que no es reducible a propiedades presentes of futuras genuinamente ocurrentes de los subsistemas $U$ y $V$. Nótese que, según la mecánica cuántica, $c^{\perp}$ no es una mera descripición de la presencia de propiedades condicionadas a que algo ocurra, sobre la base de propiedades ocurrentes de los subsistemas. Según la mecánica cuántica, la información adicional acerca de lo que es el caso está objetivamente ausente. El estado inicial $[a, X)$ incluye información acerca de propiedades disposicionales que no se reduce a información acerca de propiedades ocurrentes de los subsistemas.

$U$ podría estar en Alfa Centauro y $V$ en la Tierra cuando hacemos la medición de $S$ en $U$. Sólo entonces $d$ o $e$ serán el caso y, por lo tanto, sólo entonces estará determinado el valor de $M$ en $V$. Vemos que esta situación típica de un experimento del tipo EPR es consecuencia de la presencia de propiedades genuinamente disposicionales como la ejemplificada arriba.

$\mathrm{El}$ estado de propiedades genuinamente ocurrentes (en $t$ ) en nuestro ejemplo está representado por $[a, A)$. Las disposiciones del sistema las podemos representar por un diagrama de acceso:

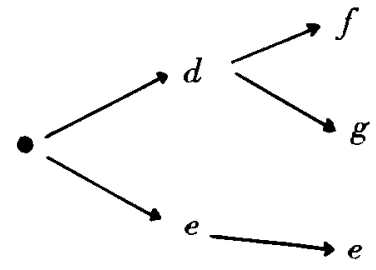

el elemento a la izquierda, $a$ en este caso, describe el estado inicial que queremos tomar convencionalmente como punto de 
partida del diagrama. Cada columna describe los estados posibles a los que el estado anterior (a la izquierda) tiene acceso. Nótese que $c$ no es accesible a partir de $a$ ya que $c$ excluye $a$ por ser ambos valores de $X$. Cada una de las transformaciones posibles (representadas por flechas) hace que cierto conjunto de propiedades genuinamente disposicionales se expresen parcialmente en términos de propiedades ocurrrentes futuras. Sin embargo, lo importante es que estas disposiciones no son reducibles a una base categorica (de propiedades ocurrentes de los subsistemas), como lo afirma la presuposición merológica newtoniana. ${ }^{6}$

4. En conclusión. hemos visto en qué sentido la nresunnsirión cómo la relación cuántica entre subsistemas es el resultado de la presencia de propiedades disposicionales genuinas. Nuestra manera de derivar esta conclusión es bastante general y sugiere que la distinción entre tipos de propiedades es una característica importante de la estructura de sistemas físicos que rechazan la presuposición merológica newtoniana.

\section{REFERENCIAS}

1989 R. Healey, The Philosophy of Quantum Mechanics, Cambridge Univ. Press, Cambridge.

1985 S. Kochen, "A New Interpretation of Quantum Mechanics" en Symposium on the Foundations of Modern Physics, P. Lahti y P. Mittelstaedt (comps.), Teaneck, N. J., World Scientific Publishing, pp. 151-70.

1988 S. Martínez, "Mediciones ideales en la mecánica cuántica", Crítica 60, dic. 1988.

6 Puede mostrarse fácilmente que el diagrama es "consistente" en el sentido de que si el estado inicial es $a$, y el sistema entra en una interacción que actualiza la situación $Z$, exactamente los mismos estados quedan accesibles a partir de $a$, que los que quedarían accesibles si el sistems entrara primero a la situación $Y$ y posteriormente a la situación $Z$. 
1990 S. Martínez, "From Mechanisms to non-separability", en preparación.

1991 S. Martínez, "Luders rule as a description of individual state transformations", to appear in 1991 in Philosophy of Science.

1989 R. Penrose, The Emperor's New Mind, Oxford Univ. Press, Oxford.

1985 R. Rosen, "Organisms as causal systems that are not mechanisms", in Theoretical Biology and Complexity, R. Rosen (comp.), Academic Press.

Recibido: 22 actubre 1990. 


\section{SUMMARY}

A central metaphysical thesis of modern science has been the idea that the structure of a physical system can be explained in terms of the properties of its constitutive subsystems. I call this presupposition the Newtonian merological presuppposition. After some brief introductory remarks on the role of this presupposition in the methodology of modern physics, and after mentioning some recent challenges to it, I focus my attention on quantum systems. Quantum mechanics is the only highly confirmed theory in which the Newtonian merological presupposition is denied. I argue that the presence of a non-Newtonian (holistic) merological structure is the result of the existence of two different types of properties, and in particular of the existence of genuinely dispositional properties. Genuinely dispositional properties are properties of a system which are not reducible to ocurrent properties of the subsystems. This distinction between two different types of properties can be made precise in a lattice theoretical modelling of the possible properties and states attributable to a quantum system. I conclude by giving an example of the sort of genuinely dispositional properties that are constitutive of quantum systems. 